\title{
Targeted gene correction and functional recovery in achondroplasia patient-derived iPSCs
}

\author{
Huan Zou ${ }^{1, *}$, Mingfeng Guan ${ }^{1, *}$, Yundong Li ${ }^{1}$, Fang Luo ${ }^{1}$, Wenyuan Wang ${ }^{1, \neq}$, Yiren Qin ${ }^{1,2, ~}$
}

${ }^{1}$ Interdisciplinary Research Center on Biology and Chemistry, Shanghai Institute of Organic Chemistry, Chinese Academy of Sciences, Shanghai 201210, China.

2State Key Laboratory of Oncogenes and Related Genes, Renji-Med X Clinical Stem Cell Research Center, Renji Hospital, School of Medicine and School of Biomedical Engineering, Shanghai Jiao Tong University, Shanghai 200127, China.

${ }^{*}$ These authors contributed equally: Huan Zou, Mingfeng Guan

łCorrespondence: Wenyuan Wang (wywang@sioc.ac.cn) or Yiren Qin (yirenqin@hotmail.com)

\begin{abstract}
Achondroplasia $(\mathrm{ACH})$ is the most common genetic form of dwarfism and belongs to dominant monogenic disorder caused by a gain-of-function point mutation in the transmembrane region of FGFR3. Stem cells and gene-editing technology provide us with effective methods and ideas for $\mathrm{ACH}$ research and treatment. Here we generated non-integrated iPSCs from an ACH girl's skin and an ACH boy's urine. We found that the chondrogenic differentiation ability of ACH iPSCs was confined compared with that of healthy iPSCs. When the mutation of ACH iPSCs was precisely corrected by CRISPR-Cas9, their chondrogenic differentiation ability could be restored. Furthermore, these corrected iPSCs displayed pluripotency, maintained normal karyotype, and demonstrated none of off-target indels. Our study may provide an important theoretical and experimental basis for the $\mathrm{ACH}$ research and treatment.
\end{abstract}

\section{Introduction}

Achondroplasia (ACH), the most common genetic form of short-limb dwarfism, is an autosomal dominant monogenic disorder (MGD) caused by a gain-of-function point mutation in the transmembrane region of fibroblast growth factor receptor 3 (FGFR3). Currently there are two mutation sites reported - Gly380Arg and Gly375Cys, and the former occupies a vast majority of ACH patients. ${ }^{1,2}$ Because homozygous ACH patients show much more severe symptoms and rarely survive, ${ }^{3,4}$ almost all survivors are heterozygous patients. The estimated frequency of ACH is 1 in 25,000 (Male 1/15000), with at least 80\% of the cases being sporadic. ${ }^{5}$ Like many other MGDs, there are no effective treatments for ACH even though the mutant gene has been identified for many years ${ }^{1}$. Fortunately, stem cell research provides potential treatments for ACH. Patient-derived stem cells can aid scientists in the investigation of specific molecular mechanisms and in the discovery of new drugs. ${ }^{6,7}$ In addition, via powerful genome editing tools, such as clustered regulatory interspaced short palindromic repeat (CRISPR)-Cas9 system, ${ }^{8}$ mutation of ACH stem cells can 
be corrected in vitro. Through safety assessment in animal models in vivo, stem cell transplantation may provide a novel therapy. ${ }^{6}$ For example, recently Perlingeiro and his colleagues used limb girdle muscular dystrophy type $2 \mathrm{~A}$ patient-derived iPSCs which were caused by mutations in the Calpain 3 (CAPN3) to perform gene correction by CRIPR-Cas9, and transplanted gene-corrected myogenic progenitors into a mouse model that combined immunodeficiency with a lack of CAPN3. They found that the transplantation corroborated the rescue of CAPN3 mRNA. ${ }^{9}$

In this study, we isolated and cultured skin, urine, and white adipose-derived somatic cells from three ACH patients with Gly380Arg mutation, including a girl, a boy, and an adult male. Further we generated non-integrated iPSCs from the ACH girl's skin and the ACH boy's urine. However adipose-derived mesenchymal stem cells (AD-MSCs) from the adult male could not be reprogrammed to iPSCs. We found that the chondrogenic differentiation ability of ACH iPSCs was confined compared with that of healthy iPSCs. When the mutation of ACH iPSCs was precisely corrected by CRISPR-Cas9, their chondrogenic differentiation ability was restored. In addition, these corrected iPSCs kept pluripotency and maintained normal chromosomal number and structure. Our study may provide an important theoretical and experimental basis for the ACH research and treatment.

\section{Materials and Methods}

\section{Research subjects and ethics statement}

Research subjects in this study included two children and one adult male. Skin and urine samples were obtained from the children. Liposuction was performed on the adult male. All human subject protocols were reviewed and approved by the Ethical Review Board of the Renji Hospital, Shanghai. All subjects signed the informed consent.

\section{Isolation and culture of SFs}

Skin biopsies were performed on the locally anesthetized lower legs by a sterile $3 \mathrm{~mm}$ skin punch. The skin tissues were cut into smaller pieces and placed in a 6-well plate, and grew for 2 weeks in the fibroblast medium that included DMEM, 10\% fetal bovine serum (FBS), penicillin/streptomycin (P/S) and glutamine. Above regents were all from ThermoFisher.

\section{Culture of UCs}

We followed the method established by Pei and his colleagues ${ }^{10}$ for the culture of UCs. Briefly, urine samples were collected into $50 \mathrm{ml}$ tubes and centrifuged at $400 \mathrm{~g}$ for 10 minutes. The supernatants were sucked out carefully, with only about $1 \mathrm{ml}$ at the bottom kept in the tube. The remaining, after being resuspended by PBS which contained amphotericin B (Selleck) and P/S, were centrifuged again. Thereafter, with the supernatants discarded, the pellets were suspended and cultured into 12-well plates by the primary 
medium that contained DMEM, Ham's F-12 (ThermoFisher), 10\% FBS, REGM Renal Epithelial Cell Growth Medium SingleQuots Kit (Lonza), amphotericin B and P/S. After 4 days, the medium was carefully changed to proliferation medium (Lonza REBM BulletKit).

Isolation and culture of AD-MSCs

Adipose biopsy was carried out by liposuction. Isolation and culture of AD-MSCs were performed according to our previous report. ${ }^{11}$ Briefly, adipose tissues were digested by $0.1 \%$ type I collagenase (Sigma) in PBS on a shaker at $37^{\circ} \mathrm{C}$ for 1 hour. Cell suspensions were then centrifuged and the pellet at the bottom was SVF. Finally, the SVF was suspended and cultured in the fibroblast medium.

\section{PCR and Sanger Sequencing}

Total genomic DNA were extracted from patient-derived peripheral blood, SFs, UCs, AD-MSCs and iPSCs by using the Genomic DNA Extraction Kit (TaKaRa). PCR was performed by using Q5 HighFidelity DNA Polymerase (New England Biolabs, NEB). FGFR3 primers were

Forward: 5'-AGGAGCTGGTGGAGGCTGA-3', Reverse: 5'-GGAGATCTTGTGCACGGTGG-3'.

PCR reactions were then purified by using GeneJET Gel Extraction Kit (ThermoFisher) and sequenced by the Genewiz.

\section{Generation of non-integrated iPSCs}

Inductions of iPSCs from SFs, UCs and AD-MSCs were performed by CytoTune iPS 2.0 Sendai Reprogramming Kit (ThermoFisher). Briefly, approximately 200,000 donor cells were cultured in a 6-well plate and transfected with the Sendai virus two days later. Twenty-four hours after transfection, the media for somatic cells were replaced with fresh ones every other day. On day 8 after transfection, the cells were digested by trypsin and 5000 cells were seeded into a 6-well plate to culture. Thereafter, the media were replaced by E8 (StemCell) every day. Three to four weeks after transfection, single cell colonies were picked up for expansion and characterization.

\section{Chondrogenic differentiation of iPSCs}

This method referred to a report of Tsumaki laboratory ${ }^{7}$. When iPSCs formed high-density cell colonies in E8, the media were changed to the initial chondrogenic medium that included DMEM/F12 (ThermoFisher), $10 \mathrm{ng} / \mathrm{ml}$ of Wnt3A (R\&D), $10 \mathrm{ng} / \mathrm{ml}$ of Activin A (Peprotech), 1\% ITS (ThermoFisher) and 1\% FBS (ThermoFisher). On day 3 after the chondrogenic induction, the medium was changed to the basal medium that contained DMEM (ThermoFisher), 1\% ITS, 1\% FBS, 2 mM L-glutamine, non-essential amino acids, $1 \mathrm{mM}$ Napyruvate (ThermoFisher), $50 \mu \mathrm{g} / \mathrm{ml}$ ascorbic acid (Sigma), $10 \mathrm{ng} / \mathrm{ml}$ BMP2 (Peprotech), 10 ng/ml TGF $\beta 1$ (Peprotech) and 10 ng/ml GDF5 (Peprotech). From day 3 to day 14, 10 ng/ml 
bFGF (Peprotech) was added to the chondrogenic medium. After Day 42, the medium was changed to the fibroblast medium.

AP staining

AP staining was performed by Vector Blue AP Substrate Kit (Vector). The procedure was conducted in accordance with the instruction.

\section{Design of sgRNAs and construction of vector}

sgRNAs were designed by using the Guide Design Resources. Then these sgRNAs were annealed and ligated to the pSpCas9(BB)-2A-RFP plasmid which was digested by Bbs I (NEB) enzyme.

\section{Transfection of CRISPR into iPSCs}

One million iPSCs were suspended in $800 \mu \mathrm{l}$ cold PBS. Thereafter, $5 \mu \mathrm{g}$ targeting plasmid and $40 \mu \mathrm{g}$ ssODNs were added into them. The cells were electroporated by using the Human Stem Cell Nucleofector Kit 2 (Lonza) and the Nucleofector 2b Device (Lonza). These cells were seeded into plates by using E8 with ROCK inhibitor. Twenty-four to forty-eight hours after electroporation, about 5000 RFP positive cells were re-seeded into a $100 \mathrm{~mm}$ plate by FACS (BD Aria II). One week later, single cell colonies were picked up and expanded for sequencing analysis.

\section{Off-target effect analysis}

Eighteen potential "off-target" sites of the sgRNA used in this research were given by the Guide Design Resources. PCR products of them were sequenced for off-target effect analysis. The sequences of the primers for off-target sites were listed in supplementary Table 1.

\section{Cell immunofluorescence}

iPSCs were fixed in 4\% paraformaldehyde solution for 15 minutes at room temperature (RT). Then they were permeabilized by using $0.1 \%$ Triton X-100 in PBS for 20 minutes at RT. After they were blocked in $5 \%$ goat serum for 1 hour, the cells were incubated overnight at $4{ }^{\circ} \mathrm{C}$ by primary antibodies, including Nanog (Abcam), SSEA-1 (Invitrogen), Oct4 (Cell signal), SSEA-4 (ThermoFisher), Sox2 (Epitomics) and TRA1-60 (Abcam). Finally, these cells were incubated by fluorescently coupled secondary antibodies (Invitrogen) and 4',6-diamidino-2-phenylindole (DAPI; Sigma) for 1 hour at RT. Images were captured on a confocal microscope (Leica).

\section{Karyotyping}

iPSCs were cultured in E8 with $0.1 \mu \mathrm{g} / \mathrm{ml}$ colchicine (ThermoFisher) for 2 hours. After the medium being removed, they were incubated in $0.56 \%$ potassium chloride for 40 minutes, and fixed in methanol and acetic 
acid (3:1 in volume) overnight. The cell suspensions were dropped onto cool slides and stained with Giemsa (ThermoFisher) for 15 minutes. More than 15 metaphase spreads of each sample were analyzed.

\section{RNA extraction and RT-qPCR}

Total RNAs were extracted from cells by using TRIzol RNA Isolation Reagents (Thermofisher). cDNAs were synthesized from total RNA with a Hifair II 1st Strand cDNA Synthesis Kit (Yeasen) for qPCR. The qPCR procedure was carried out according to the kit instructions (TB Green Premix Ex Taq kit, TakaRa). Primers used were as follows:

\section{SOX9 F: AGACCTTTGGGCTGCCTTAT \\ R: TAGCCTCCCTCACTCCAAGA}

COL2Al F: TTTCCCAGGTCAAGATGGTC

R: CTTCAGCACCTGTCTCACCA

ACAN F: AGGCAGCGTGATCCTTACC

R: GGCCTCTCCAGTCTCATTCTC

$\beta$-ACTIN F: TGGCACCACACCTTCTACAATGAGC

R: GCACAGCTTCTCCTTAATGTCACGC

\section{EdU Staining}

BeyoClick EdU-594 kit (Beyotime) was used for Edu staining. Briefly, cells were incubated in the $10 \mu \mathrm{M}$ EdU medium for 24 hours at $37^{\circ} \mathrm{C}$. After EdU solution was removed, the cells were washed twice in PBS and were used to perform the immunocytochemistry procedure.

\section{Safranin O staining}

Safranin O staining Kit (ScienCell Research Laboratories, Inc. \#8384) was used to perform this experiment. The procedure was in accordance with the instruction.

\section{Results}

\section{Identification, isolation and culture of somatic cells from $\mathrm{ACH}$ patients}

We recruited three ACH patients, including an 8-year-old girl, a 7-year-old boy and a 37-year-old adult male. Genetic mutations of the donors were confirmed by Sanger sequencing, and they are all heterozygous mutations of Gly380Arg (Fig. 1a). In addition, their clinical symptoms are consistent with the NIH-defined characteristics, such as short arms and legs, enlarged head, and prominent forehead. We punched skin tissue and collected urine from the children to culture skin fibroblasts (SFs) and urine-derived cells (UCs). Via liposuction, we obtained adipose tissue from the adult male to culture AD-MSCs. After the small pieces of skins were placed and cultured in the dish for 2 weeks, SFs gradually climbed out to proliferate (Fig. 1b, c). After the urine-derived pellets were cultured for one week, UCs formed colonies and showed epithelial 
cell morphology (Fig.1d, e). After the stromal vascular fraction (SVF) from adipose tissue was seeded and cultured for 2-3 days, AD-MSCs grew out and exhibited a typical fibroblast-like morphology (Fig. 1f, g).

\section{Generation of non-integrated iPSCs from $\mathrm{ACH}$ patients}

To generate non-integrated iPSCs from ACH patients, we used Sendai virus to transfect somatic cells. Three weeks after transfection of SFs and UCs, lots of ES cell-like colonies appeared (Fig. 2a, b). After single colonies were picked up and expanded, iPSC lines from the girl's skin (GF) and the boy's urine (BU) were established (Fig. 2c, d). These iPSCs expressed pluripotent proteins, including NANOG, OCT4, SOX2, SSEA-4 and TRA1-60, and did not express SSEA-1 (Fig. 2g). They also stained positive for the alkaline phosphatase (AP) activity (Fig. 2h). Moreover, karyotyping analysis indicated that these iPSCs maintained normal chromosomal number and structure (Fig. 2i). However, it was surprised that iPSCs could not be generated from AD-MSCs. Although small and unhealthy colonies appeared from AD-MSCs (Fig. 2e), they gradually became apoptotic and eventually died (Fig. 2f).

\section{Chondrogenic differentiation capability of ACH iPSCs}

To confirm whether the mutations would affect the function of ACH iPSCs, we performed the chondrogenic differentiation capability detection. We induced ACH iPSCs and healthy human iPSCs respectively into chondrogenic tissues by a multi-step method (Fig. 3a). We found that the chondrogenic clusters from ACH iPSCs were less and smaller than those from healthy iPSCs (Fig. 3b). By Safranin O staining, we discovered that the cartilage tissues derived from ACH iPSCs showed fewer positive areas and lower cartilage density than those from healthy iPSCs (Fig. 3c). These results suggested that the cartilage tissues derived from ACH iPSCs could produce less cartilaginous extracellular matrix. RT-qPCR results also exhibited that cartilage tissues derived from ACH iPSCs expressed lower chondrocyte-specific genes than those from healthy iPSCs, including SOX9, COL2A1 and ACAN (Fig. 3d). Our results verified that the chondrogenic differentiation capability of ACH iPSCs was confined compared with that of healthy iPSCs.

\section{Gene correction of ACH iPSCs by CRISPR-Cas9}

To perform the CRISPR-Cas-based gene correction, we designed single guide RNAs (sgRNAs) around the point mutation site of FGFR3 and a single-stranded oligo DNA nucleotides (ssODNs) homology arm donor which contained 131 nucleotides and the point mutation site (Fig. 4a). We transfected CRISPR-sgRNAs and ssODNs into ACH iPSCs, and 24-48 hours later detected the transfection efficiency by FACS. The ratio of RFP positive cells was $3.6 \%$ (Fig. $4 \mathrm{~b}$ ). After analyzing more than 100 RFP positive single cell colonies from skin, we found one completely corrected cell line. Similarly, among more than 100 analyzed RFP positive single cell colonies from urine, we also just found one completely corrected cell line (Fig. 4c). The total efficiency was less than $1 \%$. We found that corrected iPSCs expressed pluripotent proteins, 
including NANOG, OCT4, SOX2, SSEA-4 and TRA1-60, did not express SSEA-1 (Fig. 4d), and stained positive for the AP activity (Fig. 4e). The karyotyping analysis verified that corrected iPSCs maintained normal chromosomal number and structure (Fig.4f), and the Sanger sequencing showed that there were no off-target indels in them.

\section{Functional recovery of corrected ACH iPSCs}

Through morphological observation, we found that the number of chondrogenic clusters from corrected ACH iPSCs obviously increased compared with that from uncorrected cells (Fig. 5a). Via Safranin O staining, we found that there were more positive areas in cartilage tissues from corrected ACH iPSCs than those from uncorrected ones (Fig. 5b). EdU cell proliferation assay displayed that corrected cells possessed higher positive radio than uncorrected ones (Fig. 5c). RT-qPCR results also revealed that, compared with cartilage tissues from uncorrected ACH iPSCs, those from corrected ACH iPSCs expressed higher chondrocyte-specific genes - SOX9, COL2A1, and ACAN (Fig. 5d). These results suggested that the chondrogenic differentiation ability of corrected ACH iPSCs was restored.

\section{Discussion}

The average life expectancy for an $\mathrm{ACH}$ patient was decreased by 15 years compared with that for US population ${ }^{5}$. Moreover, the social concern for this disease is very low. At present there are no effective therapeutic methods for ACH. Fortunately, rapid development of stem cell biology and gene-editing technology provide a promising future for the research and treatment of $\mathrm{ACH}$ and other $\mathrm{MGDs}^{6}$.

In this study we firstly collected three different tissues from $\mathrm{ACH}$ patients to culture somatic cells, including the most commonly used skin, the most easily available urine, and a large amount of acquired adipose tissue that can produce multipotent MSCs with chondrogenic differentiation ability. We found that, like skin cells, ACH patient urine-derived cells could be efficiently reprogrammed into iPSCs, which may provide new donor cells for research of MGDs. However, to our surprise, ACH patient-derived AD-MSCs could not be reprogrammed into iPSCs. In fact, previously reported studies ${ }^{12,13}$ and our unpublished results indicated that healthy human AD-MSCs could be reprogrammed to iPSCs more efficiently than skin cells. Given that $\mathrm{ACH}$ is a regeneration dysfunction disorder of MSC-derived chondrocyte caused by FGFR3 mutation, our results suggested that perhaps the point mutation affected the reprogramming ability of ADMSCs.

Our initial hypothesis was that the point mutation might affect the chondrogenic differentiation ability of ACH iPSCs. Indeed, our experimental results confirmed that the chondrogenic differentiation ability of $\mathrm{ACH}$ iPSCs was confined compared with that of healthy iPSCs. When we used the CRISPR-Cas9 technology to correct the mutation of ACH iPSCs, we obtained two completely corrected cell lines, one from skin and the other from urine, among more than two hundred single cell colonies. The efficiency of 
precise homology directed repair (HDR) was less than 1\%. Fortunately, we found that those corrected iPSCs still displayed pluripotency and maintained normal karyotype. The sequencing analysis of potential offtarget sites suggested that there were no off-target indels in corrected iPSCs. Finally, we detected whether the function of corrected ACH iPSCs got improved. We found that, via chondrogenic induction, EdU cell proliferation assay and RT-qPCR experiments, the chondrogenic differentiation ability of corrected ACH iPSCs was indeed restored compared with that of uncorrected cells.

\section{Conclusion}

In summary, our study provided an important foundation for the further exploration of ACH research and treatment. At present, we are constructing point mutation mouse models of $\mathrm{ACH}$. In the future, we will transplant corrected ACH patient-derived MSCs or chondrocyte precursor cells into mouse models to verify their function in vivo and explore the effect of cell therapy to $\mathrm{ACH}$. Although challenges remain, the clinical application of patient-specific stem cells will be pursued through further advances in basic research (Fig. 6).

\section{Acknowledgements}

We would like to thank Dr. Wei-Qiang Gao for his helpful discussion in conceiving the project, Dr. Hui Yang for providing pSpCas9(BB)-2A-RFP plasmid, Chikai Zhou and Dr. Erwei Zuo for their helpful discussion in designing sgRNA, Dr. Sushan Luo for skin biopsies, Guangrui Cao for her helpful language modification.

\section{Author contributions}

H.Z. and M.G. performed experiments and analyzed data, Y.L., and F.L. performed experiments; W-Y.W. provided experimental platform and financial support; Y.Q conceived and designed the study, performed experiments, analyzed data, provided financial support and wrote the manuscript.

\section{Funding statement}

This work was funded by grants from National Natural Science Foundation of China (General Program 31570992) to Y.Q., National Key R\&D Program of China (Grant No. 2018YFA0107903, 2016YFA0501902) and Shanghai Municipal Science and Technology Major Project (Grant No. 2019SHZDZX02) to W-Y.W.

\section{Conflicts of Interest}

There is no potential conflict of interest.

\section{Supplementary Material}


Supplementary data - Ethics statement

Supplemental Table S1

\section{References}

1. Shiang R, Thompson LM, Zhu YZ, et al. Mutations in the transmembrane domain of FGFR3 cause the most common genetic form of dwarfism, achondroplasia. Cell 1994;78:335-342. DOI: 10.1016/00928674(94)90302-6.

2. Superti-Furga A, Eich G, Bucher HU, et al. A glycine 375-to-cysteine substitution in the transmembrane domain of the fibroblast growth factor receptor-3 in a newborn with achondroplasia. Eur J Pediatr 1995;154:215-219. DOI: 10.1007/BF01954274.

3. Pauli RM, Conroy MM, Langer Jr LO, et al. Homozygous achondroplasia with survival beyond infancy. Am J Med Genet 1983;16:459-473. DOI: 10.1002/ajmg.1320160404.

4. JT Hecht, WA Horton, IJ Butler, et al. Foramen magnum stenosis in homozygous achondroplasia. Eur J Pediatr 1986;145:545-547. DOI: 10.1007/BF02429060.

5. Horton WA, Hall JG and Hecht JT. Achondroplasia. Lancet 2007;370:162-172. DOI: 10.1016/S01406736(07)61090-3.

6. Qin Y and Gao WQ. Concise Review: Patient-Derived Stem Cell Research for Monogenic Disorders. Stem Cells 2016;34:44-54. DOI: 10.1002/stem.2112.

7. Yamashita A, Morioka M, Kishi H, et al. Statin treatment rescues FGFR3 skeletal dysplasia phenotypes Nature 2014;513:507-511. DOI: 10.1038/nature13775.

8. Cong L, Ran FA, Cox D, et al. Multiplex genome engineering using CRISPR/Cas systems. Science 2013;339:819-823. DOI: 10.1126/science.1231143.

9. Selvaraj S, Dhoke NR, Kiley J, et al. Gene correction of Igmd2a patient-specific iPSCs for the development of targeted autologous cell therapy. Mol Ther 2019;27:2147-2157. DOI: 10.1016/j.ymthe.2019.08.011.

10. Zhou T, Benda C, Duzinger S, et al. Generation of induced pluripotent stem cells from urine. J Am Soc Nephrol 2011;22:1221-1228. DOI: 10.1681/ASN.2011010106.

11. Qin Y, Qin J, Zhou C, et al. Generation of embryonic stem cells from mouse adipose-tissue derived cells via somatic cell nuclear transfer. Cell Cycle 2015;14:1282-1290. DOI:

10.1080/15384101.2015.1007732.

12. Sun N, Panetta NJ, Gupta DM, et al. Feeder-free derivation of induced pluripotent stem cells from adult human adipose stem cells. Proc Natl Acad Sci USA 2009;106:15720-15725. DOI: 10.1073/pnas.0908450106.

13. Sugii S, Kida Y, Kawamura T, et al. Human and mouse adipose-derived cells support feederindependent induction of pluripotent stem cells. Proc Natl Acad Sci USA 2010;107:3558-3563. DOI: 10.1073/pnas.0910172106.

\section{Figures and Figure Legends}


bioRxiv preprint doi: https://doi.org/10.1101/801415; this version posted December 17, 2020. The copyright holder for this preprint (which was not certified by peer review) is the author/funder, who has granted bioRxiv a license to display the preprint in perpetuity. It is made available under aCC-BY-NC-ND 4.0 International license.

A

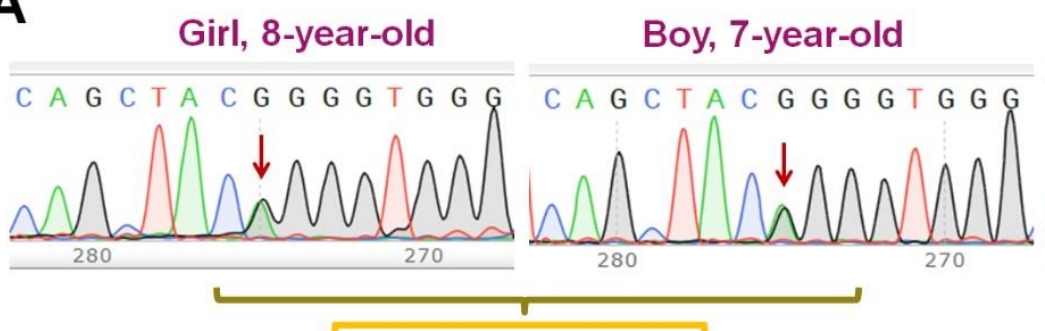

Girl, 8-year-old

Boy, 7-year-old

Man, 38-year-old
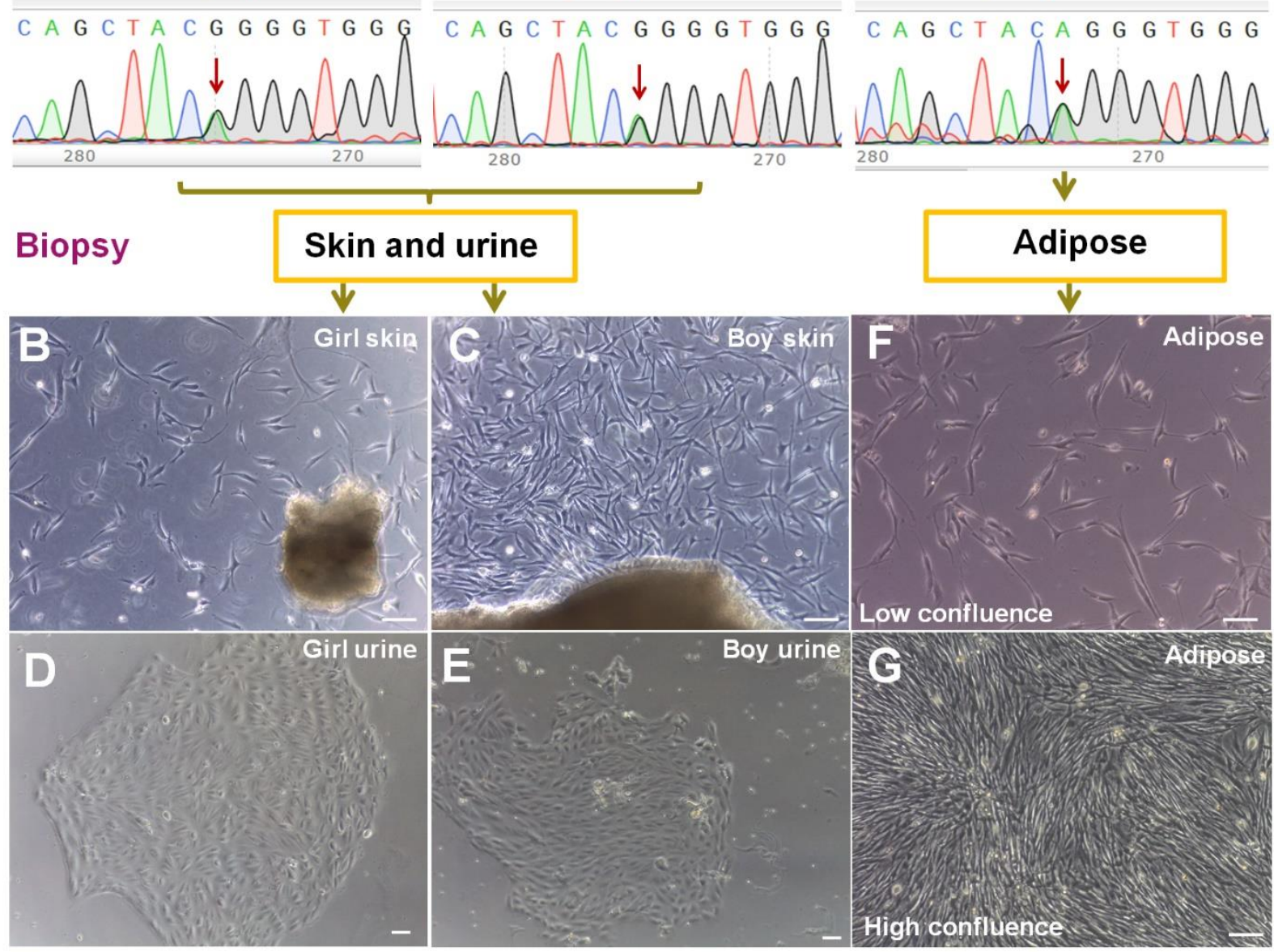

Adipose

$\downarrow$
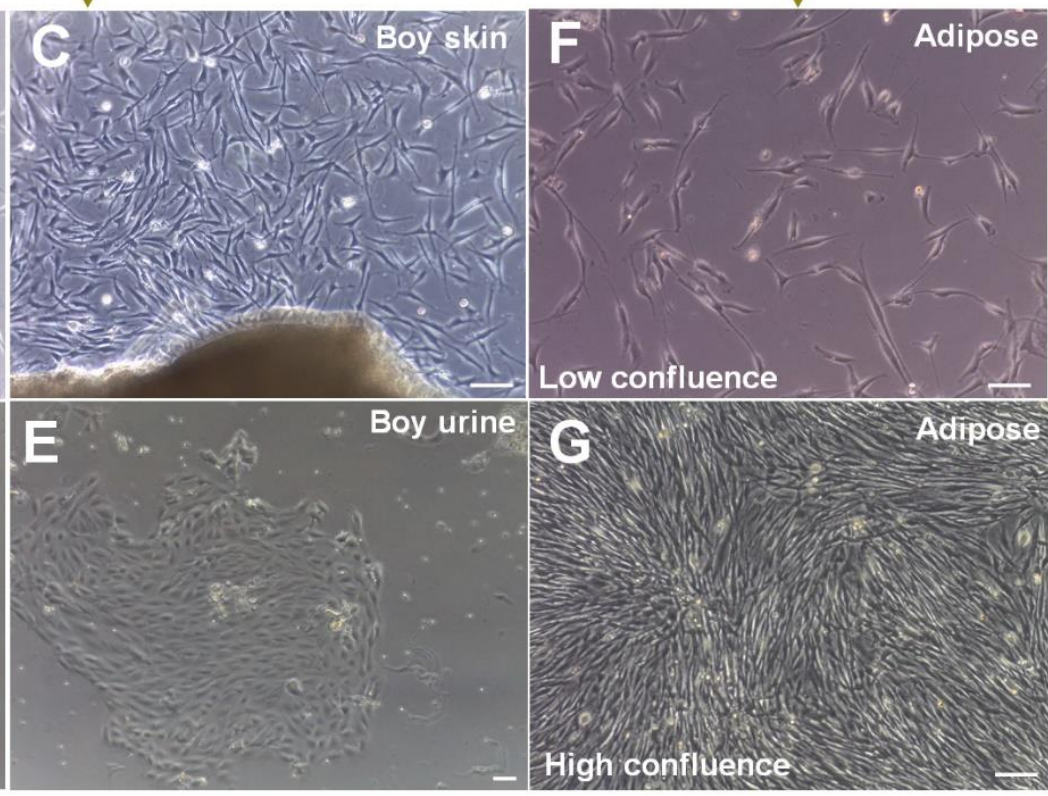

FIG. 1. Identification, isolation and culture of somatic cells from $\mathrm{ACH}$ patients. (A) DNA sequences of the three ACH patients showed that these disorders were all caused by heterozygous mutations of Gly380Arg in the FGFR3. (B, C) SFs from the girl and the boy. (D, E) UC colonies from the girl and the boy showed epithelial cell morphology. (F, G) AD-MSCs from the adult male exhibited a typical fibroblast-like morphology. Bar in all panels: $10 \mu \mathrm{m}$. 

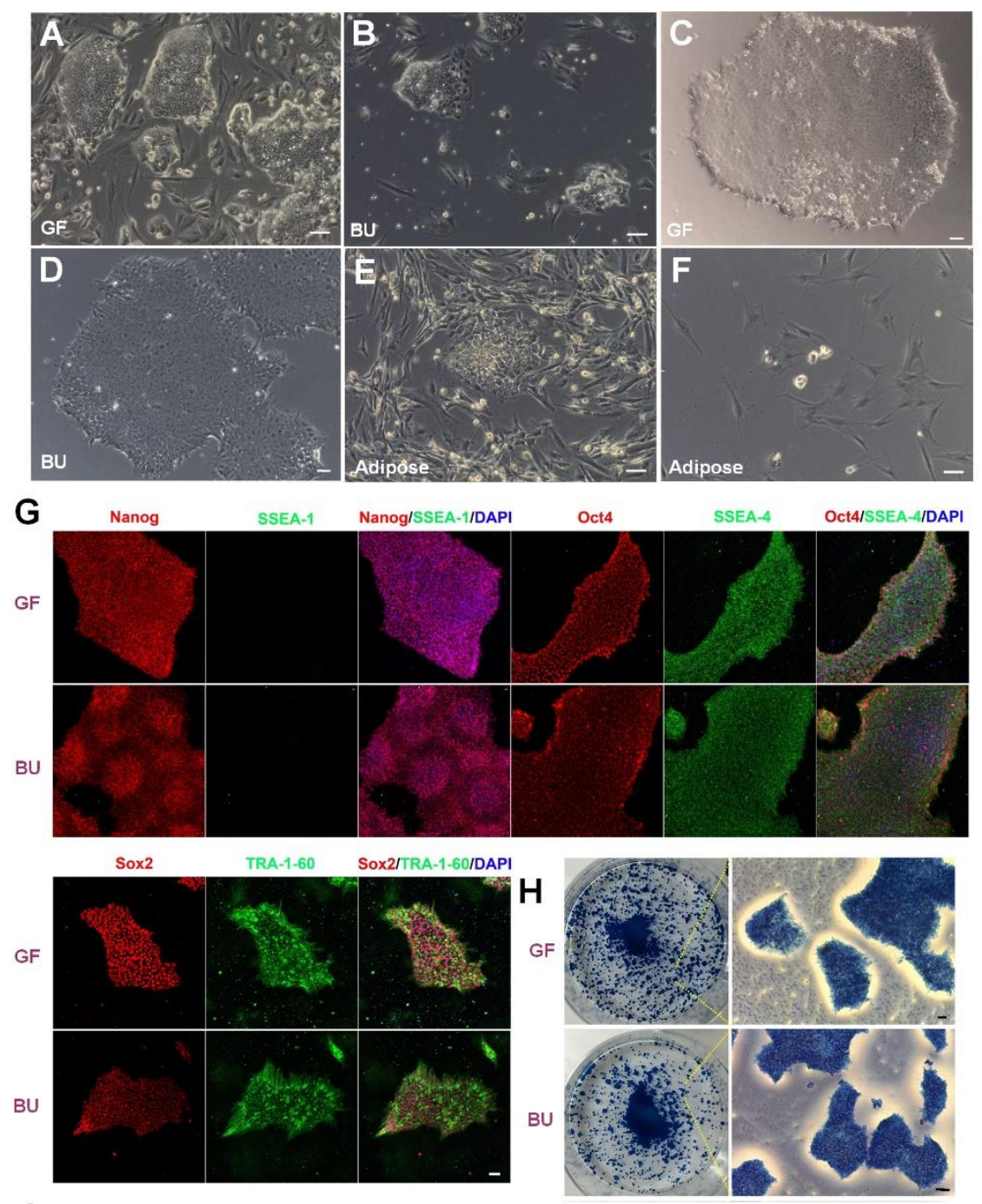

I

GF

BU
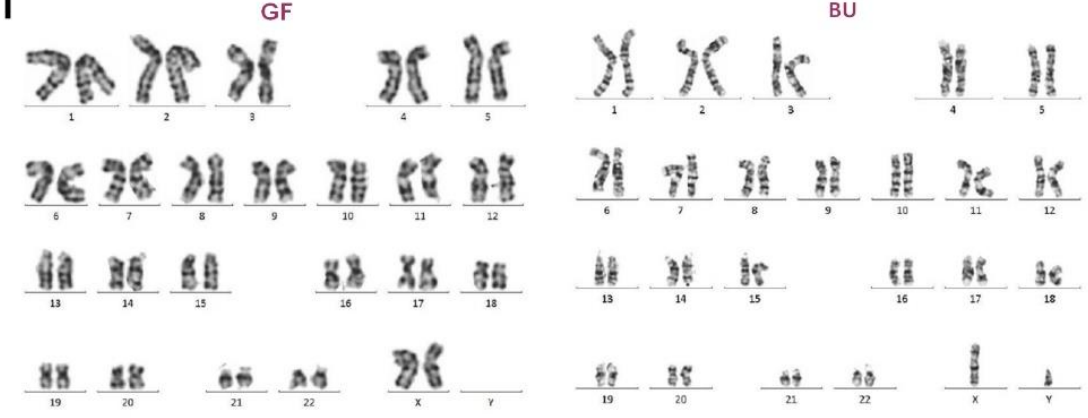

FIG. 2. Generation and characterization of non-integrated iPSCs from ACH patients. (A, B) 3 weeks ES cell-like colonies from the girl's SFs (GF) and the boy's UCs (BU) after transfection. (C) Expanded iPSCs from GF. (D) Expanded iPSCs from BU. (E) 3 weeks ES cell-like colonies from AD-MSCs after transfection were small and unhealthy. (F) Colonies from AD-MSCs gradually became apoptotic and eventually died. (G) iPSCs from GF and BU expressed pluripotent protein, including NANOG, OCT4, SOX2, SSEA4 and TRA1-60, and did not express SSEA-1. (H) iPSCs from GF and BU stained positive for the AP activity. (I) iPSCs from GF and BU indicated normal chromosomal number and structure. Bar in all panels: $10 \mu \mathrm{m}$. 
bioRxiv preprint doi: https://doi.org/10.1101/801415; this version posted December 17, 2020. The copyright holder for this preprint (which was not certified by peer review) is the author/funder, who has granted bioRxiv a license to display the preprint in perpetuity. It is made available under aCC-BY-NC-ND 4.0 International license.

A

A

$0 \quad 1-3$

4-42

43-60

E8 $\quad 1 \%$ ITS, $1 \%$ FBS

DMEM, $1 \%$ ITS, $1 \% \mathrm{FBS}$

$50 \mu \mathrm{g} / \mathrm{ml}$ Ascorbic acid, $10 \mathrm{ng} / \mathrm{ml} \mathrm{BMP2}$

$10 \mathrm{ng} / \mathrm{ml} \mathrm{TGF} \beta, 10 \mathrm{ng} / \mathrm{ml}$ GDF5

$10 \mathrm{ng} / \mathrm{ml}$ Activin A

$10 \mathrm{ng} / \mathrm{ml}$ bFGF (day 4-14)

Day

$0-14$

15-60

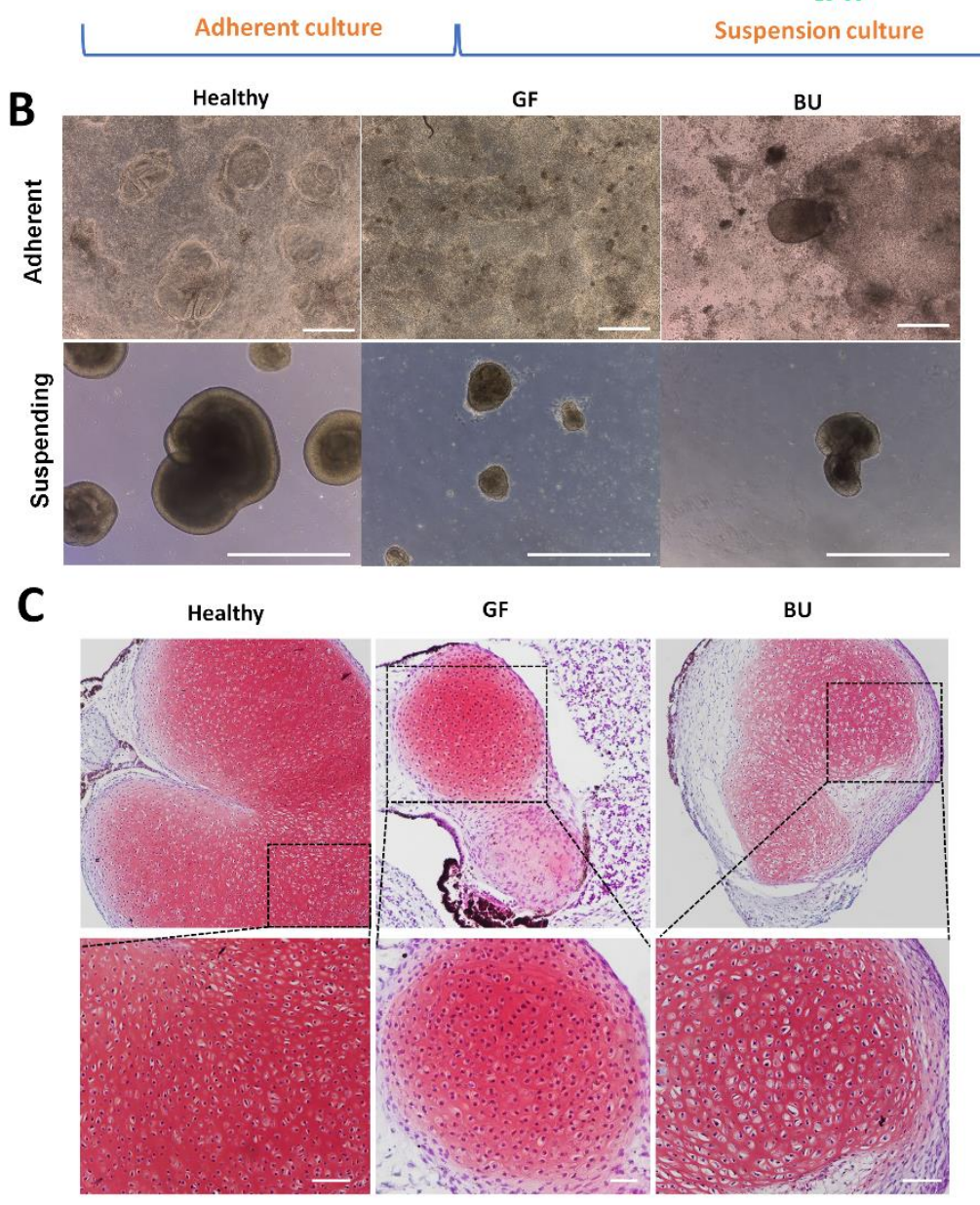

D
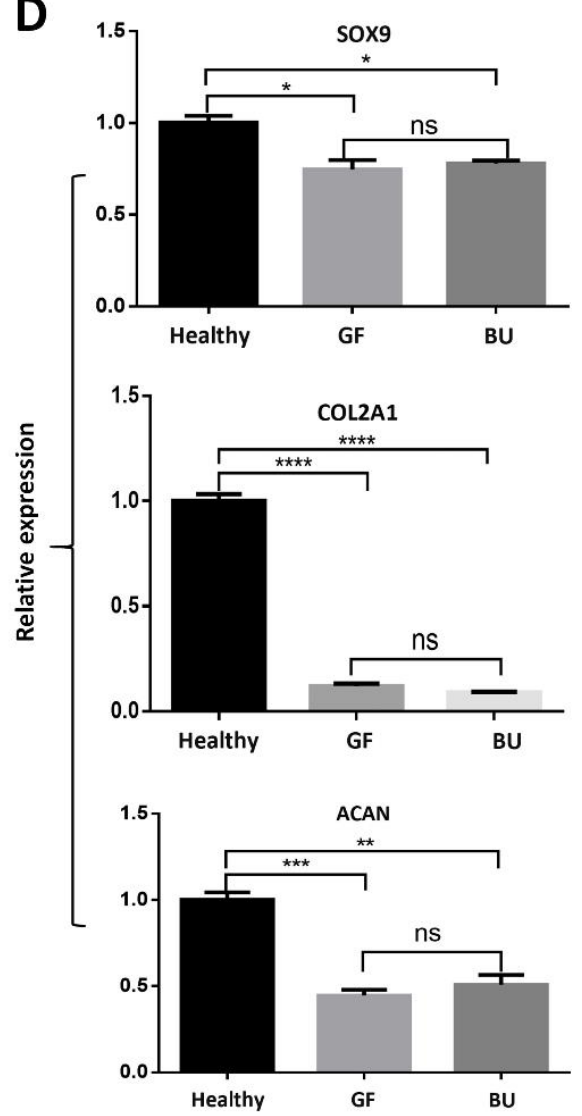

FIG. 3. Chondrogenic differentiation of ACH iPSCs. (A) A multi-step chondrogenic induction method. (B) Chondrogenic clusters from $\mathrm{ACH}$ iPSCs were less and smaller than those from healthy iPSCs. Bar: $100 \mu \mathrm{m}$. (C) Safranin O staining displayed that there were fewer positive areas and lower cartilage density in cartilage tissues from ACH iPSCs than those from healthy iPSCs. Bar: $10 \mu \mathrm{m}$. (D) RT-qPCR results also exhibited that cartilage tissues derived from ACH iPSCs expressed lower chondrocyte-specific genes, such as SOX9, COL2A1, and ACAN, than those from healthy iPSCs. Symbol "**" stands for $p<0.05$. Symbol "**" stands for $p<0.01$. Symbol "***" stands for $p<0.001$. Symbol "****" stands for $p<0.0001$. 
bioRxiv preprint doi: https://doi.org/10.1101/801415; this version posted December 17, 2020. The copyright holder for this preprint (which was not certified by peer review) is the author/funder, who has granted bioRxiv a license to display the preprint in perpetuity. It is made available under aCC-BY-NC-ND 4.0 International license.

A

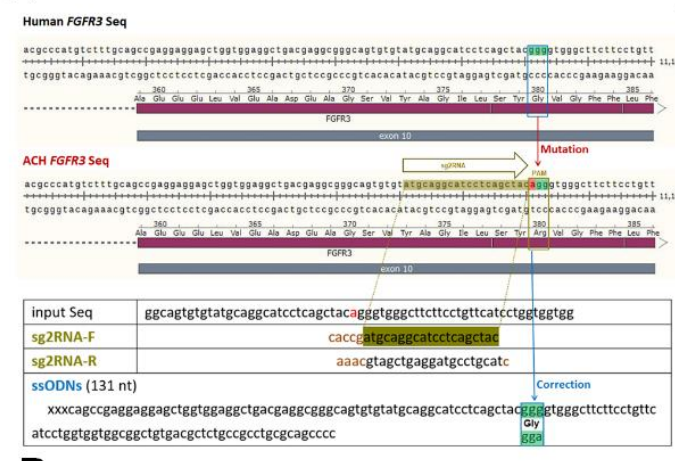

B

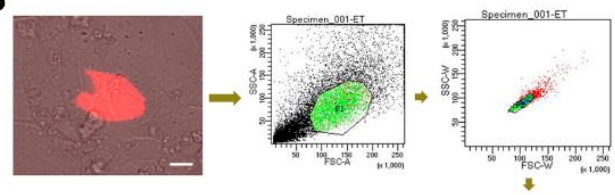

$+$

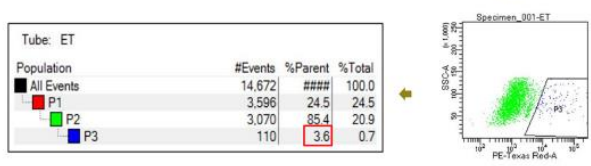

C
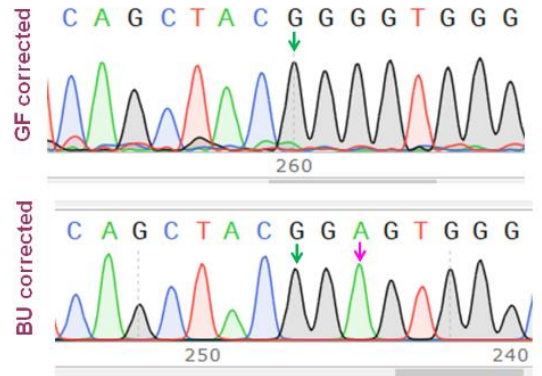

D
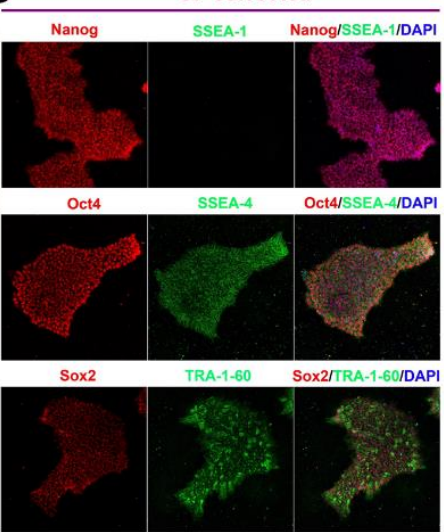

E

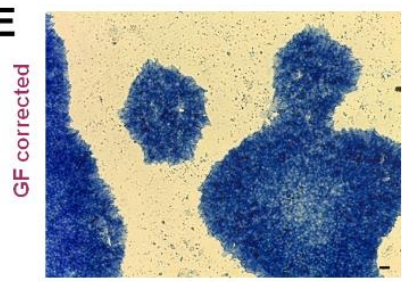

$\mathbf{F}$

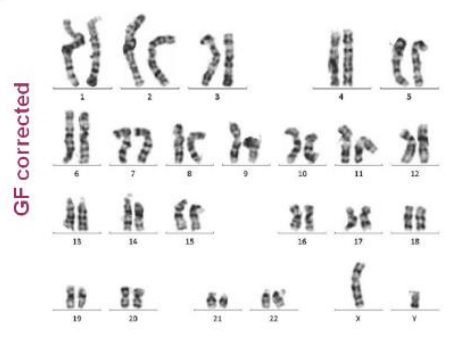

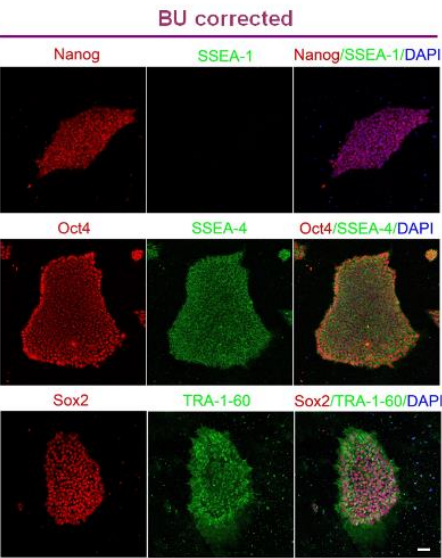
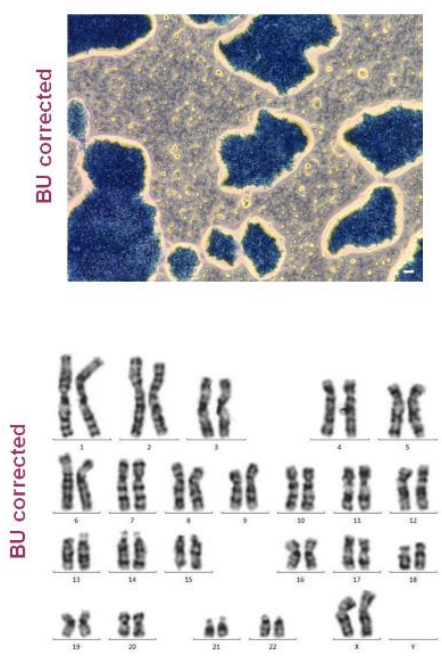

FIG. 4. Gene correction of ACH iPSCs by CRISPR-Cas9 and characterization of corrected iPSCs. (A) Designed sgRNA and ssODNs around the point mutation site. (B) FACS analysis showed that the ratio of RFP positive cells was 3.6\%. (C) Corrected iPSCs showed normal DNA sequences (Pink arrow indicated a synonymous mutation). (D) Corrected iPSCs expressed NANOG, OCT4, SOX2, SSEA4 and TRA1-60, and did not express SSEA-1. (E) Corrected iPSCs stained positive for the AP activity. (F) Corrected iPSCs showed normal chromosomal number and structure. Bar in all panels: $10 \mu \mathrm{m}$. 

under aCC-BY-NC-ND 4.0 International license.

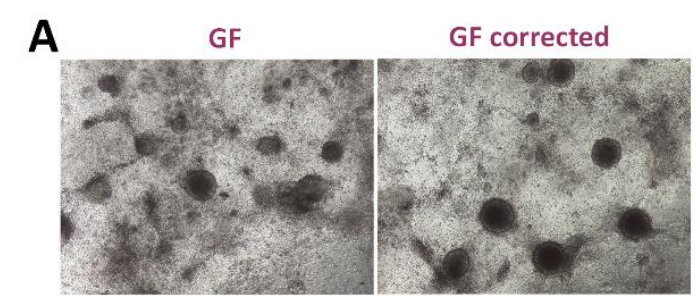

BU

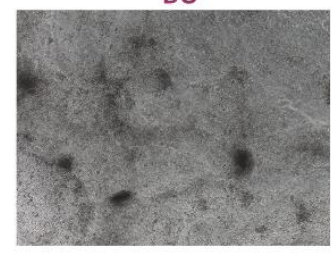

C

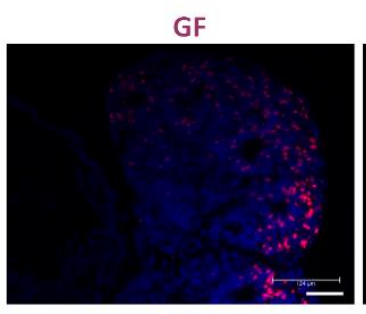

BU

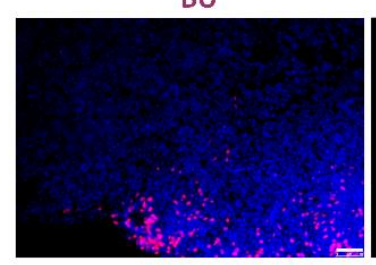

D
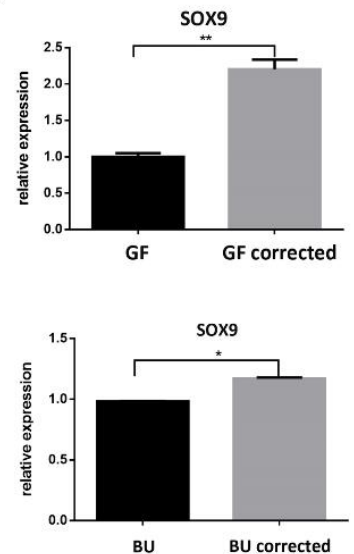

BU

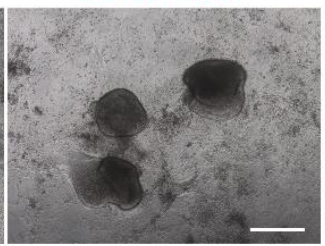

BU corrected
B GF

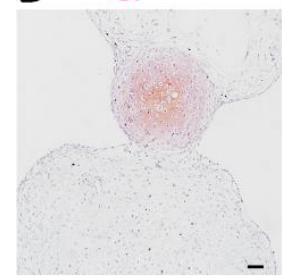

BU

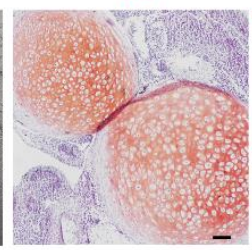

GF corrected

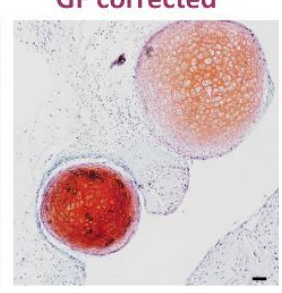

BU corrected

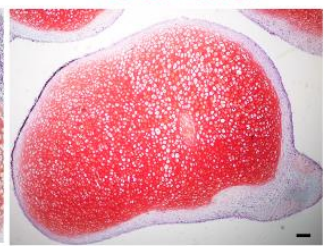

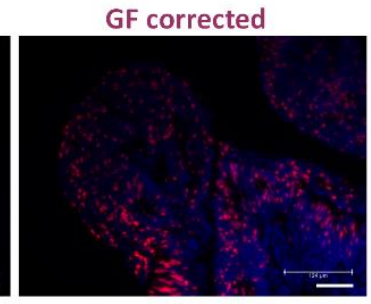

BU corrected
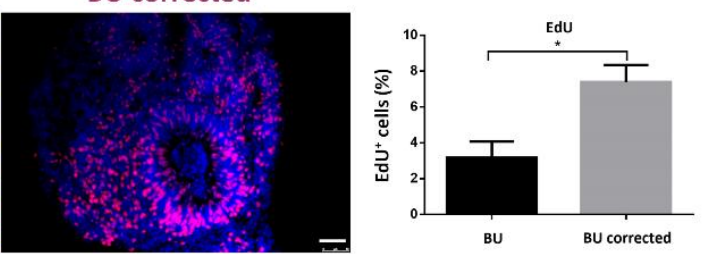

BU
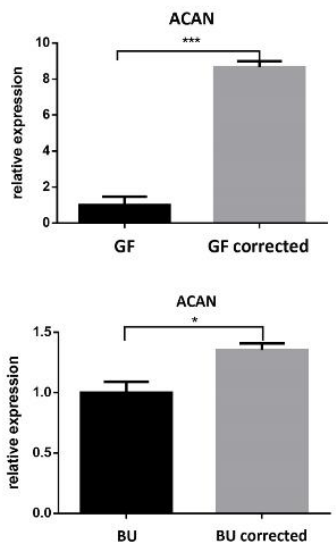

FIG. 5. Chondrogenic differentiation of corrected ACH iPSCs. (A) The number of chondrogenic clusters from corrected iPSCs obviously increased compared with that from uncorrected cells. Bar: $100 \mu \mathrm{m}$. (B) Safranin O staining illustrated that cartilage tissues from corrected ACH iPSCs demonstrated more positive areas than those from uncorrected cells. Bar: $10 \mu \mathrm{m}$. (C) EdU cell proliferation assay indicated that cartilage tissues from corrected iPSCs showed higher positive radio than those from uncorrected cells. Bar: 50 $\mu \mathrm{m}$. Symbol “*” stands for $p<0.05$. (D) RT-qPCR results also revealed that the expression level of chondrocyte-specific genes - SOX9, COL2A1, and ACAN from corrected ACH iPSCs were higher than those of ACH iPSCs. 
bioRxiv preprint doi: https://doi.org/10.1101/801415; this version posted December 17, 2020. The copyright holder for this preprint (which was not certified by peer review) is the author/funder, who has granted bioRxiv a license to display the preprint in perpetuity. It is made available under aCC-BY-NC-ND 4.0 International license.

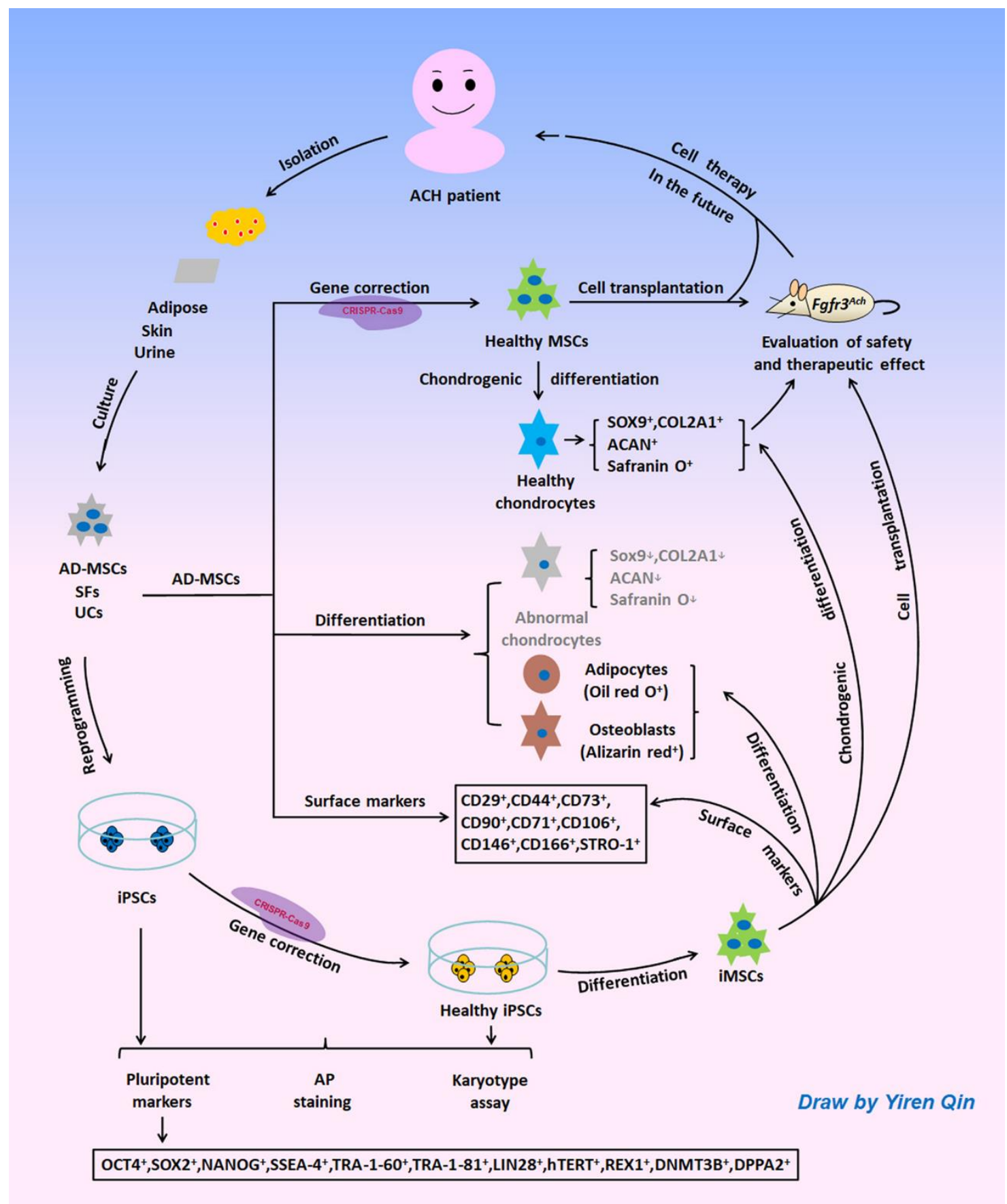

FIG. 6. Diagrammatic strategy of $\mathrm{ACH}$ stem cell research. Somatic cells can be isolated and cultured from ACH patient-derived adipose, skin, and urine, and can further be reprogrammed into iPSCs. After gene correction of iPSCs or AD-MSCs via CRISPR-Cas9, they can be differentiated into healthy cells, such as MSCs or chondrocyte precursor cells. Then these healthy cells can be transplanted into $\mathrm{ACH}$ mouse models to assess their relative safety and therapeutic effects. The clinical application of $\mathrm{ACH}$ patient-derived stem cells will be pursued in the future. 